\title{
Erratum: Unsafe abortion in rural Tanzania - the use of traditional medicine from a patient and a provider perspective
}

Vibeke Rasch ${ }^{1 *}$, Pernille H. Sørensen ${ }^{2}$, Anna R. Wang ${ }^{3}$, Flora Tibazarwa ${ }^{4}$ and Anna K. Jäger ${ }^{5}$

\section{Erratum}

The original version of this article [1] unfortunately contained a mistake. The presentation of Table 2 was incorrect in the HTML and PDF versions of this article with the following errors:

The local name 'Eyabia' has moved down a row causing subsequent rows to move out of order.

In addition, there are several errors in the reported effects of Ocimum suave, Vernonia amygdalina and Sphaerogyne latifolia.

The corrected Table 2 is given below.

\author{
Author details \\ 'Department of Obstetrics and Gynaecology, Odense University Hospital, \\ Odense C 5000, Denmark. ${ }^{2}$ Department of International Health, Immunology \\ and Microbiology, Faculty of Health and Medical Sciences, University of \\ Copenhagen, 3D Blegdamsvej, Copenhagen N 2200, Denmark. ${ }^{3}$ The Medical \\ Faculty, University of Southern Denmark, Odense C 5000, Denmark. \\ ${ }^{4}$ Department of Botany, University of Dar es Salaam, Dar es Salaam, Tanzania. \\ ${ }^{5}$ Department of Drug Design and Pharmacology, Faculty of Health and \\ Medical Sciences, University of Copenhagen, 2 Universitetsparken, \\ Copenhagen O 2100, Denmark.
}

Received: 1 December 2015 Accepted: 17 December 2015

Published online: 29 December 2015

\section{Reference}

1. Rasch V, Sørensen PH, Wang AR, Tibazarwa F, Jäger AK. Unsafe abortion in rural Tanzania - the use of traditional medicine from a patient and a provider perspective. BMC Pregnancy Childbirth. 2014;14:419. doi:10.1186/s12884-014-0419-6.

\footnotetext{
* Correspondence: vrasch@health.sdu.dk

${ }^{1}$ Department of Obstetrics and Gynaecology, Odense University Hospital, Odense C 5000, Denmark

Full list of author information is available at the end of the article
}

Submit your next manuscript to BioMed Central and we will help you at every step:

- We accept pre-submission inquiries

- Our selector tool helps you to find the most relevant journal

- We provide round the clock customer support

- Convenient online submission

- Thorough peer review

- Inclusion in PubMed and all major indexing services

- Maximum visibility for your research

Submit your manuscript at

www.biomedcentral.com/submit

\section{() Biomed Central}


Table 2 Plant species used for induction of abortion in Tanzania

\begin{tabular}{|c|c|c|c|c|}
\hline & Local name & Latin name & Effect on force of contraction & Effect on frequency of contraction \\
\hline 1 & Akakurura & Bidens pilosa $\mathrm{L}$. & ++ & 0 \\
\hline 2 & Akaramata & Rubia cordifolia & + & + \\
\hline 3 & Akashwagara & Ocimum suave Klilld. & ++ & + \\
\hline 4 & Eitezi & Commelina africana $\mathrm{L}$. & ++ & + \\
\hline 5 & Ekijumbura & Obetia radula (Baker) B.D.Jacks. & + & + \\
\hline 6 & Engenyi & Zehneria scabra (Linn. F.) Sond.E & 0 & + \\
\hline 7 & Enkaka Aloe Vera & Aloe $s p$ & 0 & + \\
\hline 8 & Eyabia & Oldenlandia corymbosa L. & ++ & 0 \\
\hline 9 & Eyabya no. 2 & Macrotyloma axillare (E.Mey.) Verdc & 0 & 0 \\
\hline 10 & Kaitampunu & Manihot esculenta Crantz & ++ & 0 \\
\hline 11 & Kikikarabo & Triumfetta microphylla Wight \& Arn & + & + \\
\hline 12 & Muarobaini & Azadirachta indica A. Juss. & 0 & + \\
\hline 13 & Omubabazi no. 1 & Desmodium barbatum (L.) Benth & ++ & + \\
\hline 14 & Omubabazi no.2 & Sphaerogyne latilifolia Naudin & ++ & + \\
\hline 15 & Omubirizi & Vernonia amygdalina Delile & 0 & 0 \\
\hline 16 & Omudimu & Citrus sinensis (L.) Osbeck & 0 & 0 \\
\hline 17 & Omujuna & Ricinus communis $\mathrm{L}$. & + & 0 \\
\hline 18 & Omushangati & Canthium sp. & 0 & + \\
\hline 19 & Omutoma & Ficus thonningii Blume & 0 & 0 \\
\hline 20 & Orwangwe & Cassia mimosoides $\mathrm{L}$. & 0 & 0 \\
\hline 21 & Webumbe & Biophytum helenae Buscal \& Muschi & 0 & + \\
\hline
\end{tabular}

